\title{
Giant magnetoimpedance in trilayer structures of patterned magnetic amorphous ribbons
}

\author{
F. Amalou ${ }^{\mathrm{a})}$ and M. A. M. Gijs \\ Institute of Microelectronics and Microsystems, Swiss Federal Institute of Technology Lausanne (EPFL), \\ CH-1015 Lausanne, Switzerland
}

(Received 9 April 2002; accepted for publication 17 June 2002)

\begin{abstract}
We have measured the giant magnetoimpedance (GMI) and giant magnetoresistance (GMR) of Metglas ${ }^{\circledR} / \mathrm{Cu} /$ Metglas trilayer stacks of micropatterned amorphous magnetic Metglas 2714a ribbons and $\mathrm{Cu}$ foils. We obtain room-temperature GMI ratios $\left[Z(0)-Z\left(H_{s}\right)\right] / Z\left(H_{s}\right)$ up to $800 \%$, and GMR ratios $\left[R(0)-R\left(H_{s}\right)\right] / R\left(H_{s}\right)$ up to $2400 \%$ at frequencies of $100 \mathrm{kHz}-10 \mathrm{MHz}$, with $H_{s}$ the magnetic field in which impedance and resistance saturate towards their minimum values. These high values are a direct consequence of the large dynamic relative permeability of the magnetic ribbons and the chosen trilayer geometry. We analyze our results in the context of a theoretical model of L. V. Panina and K. Mohri [Sens. Actuators A 81, 71 (2000)]. (C) 2002 American Institute of Physics. [DOI: 10.1063/1.1499769]
\end{abstract}

The giant magnetoimpedance (GMI) effect in ultrasoft magnetic FeCoSiB alloys is a subject of increasing interest because of its high potential for magnetic sensing applications. The GMI effect is a change in the complex impedance at high frequencies (usually $>0.1 \mathrm{MHz}$ ) and arises from the magnetic field-induced change of the dynamic relative permeability $\mu_{r}$ of the magnetic material. Also, a related highfrequency giant magnetoresistance (GMR) effect can be measured as a consequence of the magnetic field-induced change of the skin depth. Due to the extremely high value and large field-induced variation of $\mu_{r}$ for the amorphous FeCoSiB alloys, GMI effects $\left[Z(0)-Z\left(H_{s}\right)\right] / Z\left(H_{s}\right)$, of the order of $100 \%$ have been reported for both amorphous wires $^{1-4}$ and ribbons ${ }^{5-8}$ at fields $<1 \mathrm{mT}$, with $Z\left(H_{s}\right)$ the minimum value of the impedance at high fields. Recently, increased GMI and GMR effects (up to 400\%) have been observed in sputtered $\mathrm{F} / \mathrm{Cu} / \mathrm{F}$ trilayers, in which the ferromagnetic material $F$ can be $\mathrm{CoSiB}, \mathrm{NiFe}$, or FeSiB. ${ }^{9-11}$ Typically, sputtered magnetic film thicknesses are of the order of $0.1-1 \mu \mathrm{m}$.

In this letter, we report on the GMI and GMR effects of trilayer stacks of microstructured Metglas 2714a/Cu/Metglas 2714a amorphous magnetic ribbons. Metglas ${ }^{12} 20-30 \mu \mathrm{m}$ thick $^{12}$ and $\mathrm{Cu}$ foils are glued and micropatterned on glass substrates using a lithographic process. The combination of these intrinsically very high- $\mu_{r}$ materials in a trilayer configuration, in which magnetic flux closure between upper and lower ferromagnetic layers results in extremely high effective permeabilities, is reflected by very large values of the measured effects: a GMI ratio up to $800 \%$ and a GMR ratio up to $2400 \%$. We analyze the GMI and GMR as a function of sample size, frequency, and magnetic field, and interpret our results in the context of the model of Panina and Mohri. ${ }^{10}$

The inset of Fig. 1 shows a top and side view of the test structures; the outer squares are electrical contacts. Table I lists the dimension of the various samples studied. The amorphous ribbons are glued on a glass wafer and patterned using

${ }^{a)}$ Electronic mail: farid.amalou@epfl.ch a standard lithographic procedure and wet etching, resulting in an array of rectangular mm-sized structures. A $25 \mu \mathrm{m}$ thick copper foil is then glued on the magnetic structures and patterned into a 0.5 -mm-wide conductor. Finally, a third layer of amorphous magnetic foil is glued and structured, similar to the first one. We consider three trilayer samples of
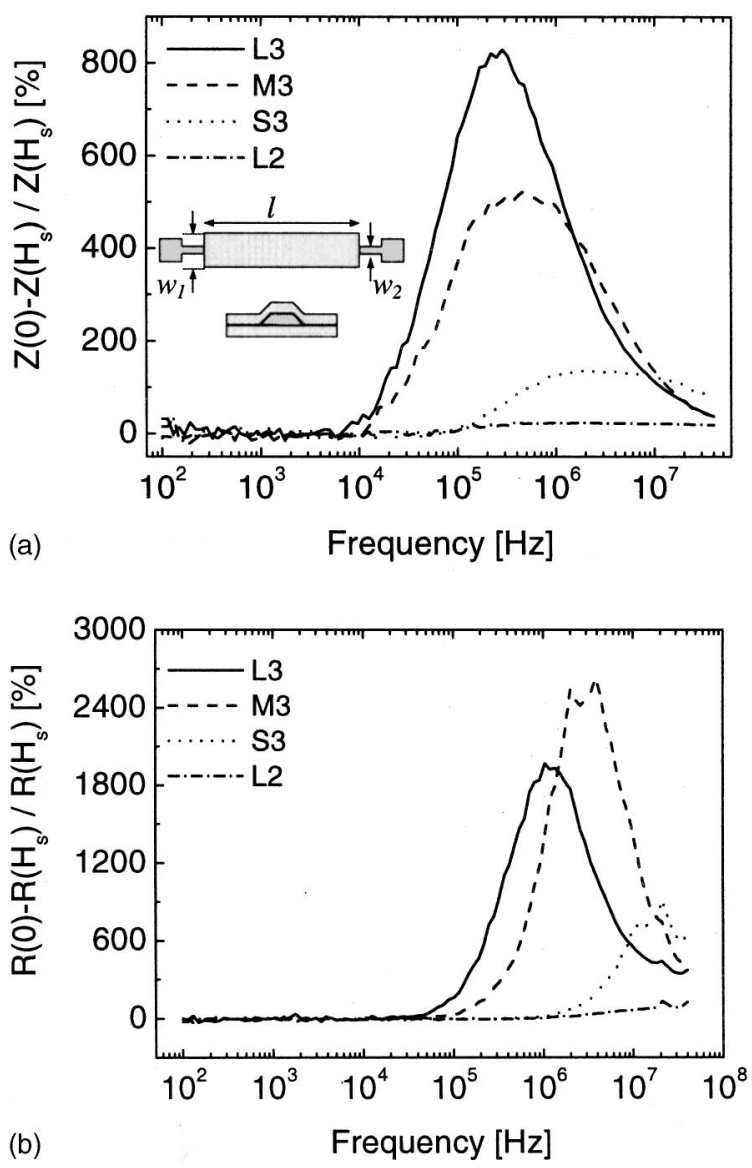

FIG. 1. Frequency dependence of (a) the giant magnetoimpedance and (b) the giant magnetoresistance ratios for the samples of Table I. The inset shows a schematic top and side view of the samples. 
TABLE I. Identification of samples studied. Length $\ell$ and widths $w_{1}$ and $w_{2}$ of the samples are defined in the inset of Fig. 1.

\begin{tabular}{cccc}
\hline \hline Sample & $\ell(\mathrm{mm})$ & $w_{1}(\mathrm{~mm})$ & $w_{2}(\mathrm{~mm})$ \\
\hline L3 & 16.0 & 3.2 & 0.5 \\
M3 & 10.0 & 2.0 & 0.5 \\
S3 & 5.0 & 1.0 & 0.2 \\
L2 & 16.0 & 3.2 & 0.5 \\
\hline \hline
\end{tabular}

different sizes (L3, M3, and S3), as well as one sample having a $\mathrm{Cu}$ layer and just the lower magnetic layer (L2). The magneto-impedance measurements are carried out in magnetic fields up to $30 \mathrm{mT}$, applied by a solenoid parallel to the long axis of the sample. The electrical characterization of the samples is performed with a HP 4194A Impedance/GainPhase Analyzer, whereby frequency is varied from $1 \mathrm{kHz}$ up to $40 \mathrm{MHz}$. Careful calibration of the measurement setup enables to distinguish the resistive and reactive parts of the impedance. The experimental setup is fully controlled by a computer using a LABVIEW interface.

Impedance and resistance have been measured as a function of magnetic field. Typical curves indicate, in the 100 $\mathrm{kHz}-1 \mathrm{MHz}$ frequency range, a strong decrease of the resistance towards a saturation value (at fields around 1-10 mT). We have plotted in Figs. 1(a) and 1(b) the frequency-dependent variation of the impedance ratio $\left[Z(0)-Z\left(H_{s}\right)\right] / Z\left(H_{s}\right)$ and resistance ratio $\left[R(0)-R\left(H_{s}\right)\right] / R\left(H_{s}\right)$, with $H_{s}$ the magnetic field, in which impedance and resistance saturate to their lower values. Very high ratios are obtained, ranging from $800 \%$ for the GMI effect to $2400 \%$ for the GMR effect. We attribute these high values to the choice of the material and the trilayer structure; indeed, sample L2, having just a single magnetic layer, has only a GMI ratio of about $30 \%$ and a GMR ratio of about $150 \%$. The trilayer geometry clearly allows a nearly perfect flux closure for the magnetic field generated by the ac current through the sample, leading to much higher GMI and GMR effects.

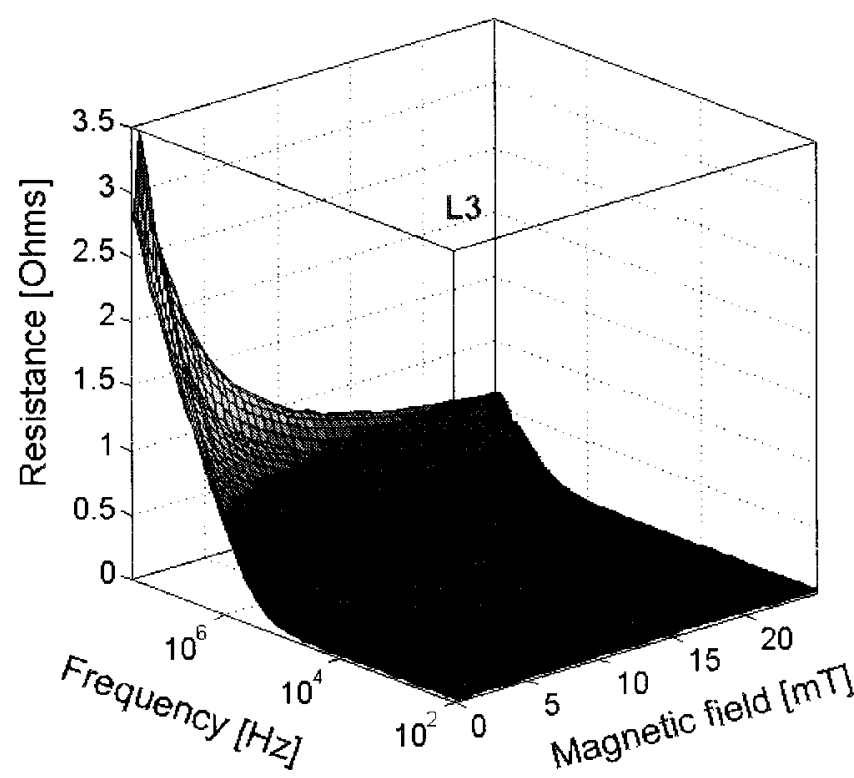

FIG. 2. Three-dimensional plot of the resistance as a function of frequency and magnetic field for the trilayer structure L3 of Table I. and magnetic field for the trilayer structure L3 of Table I. nancial support.

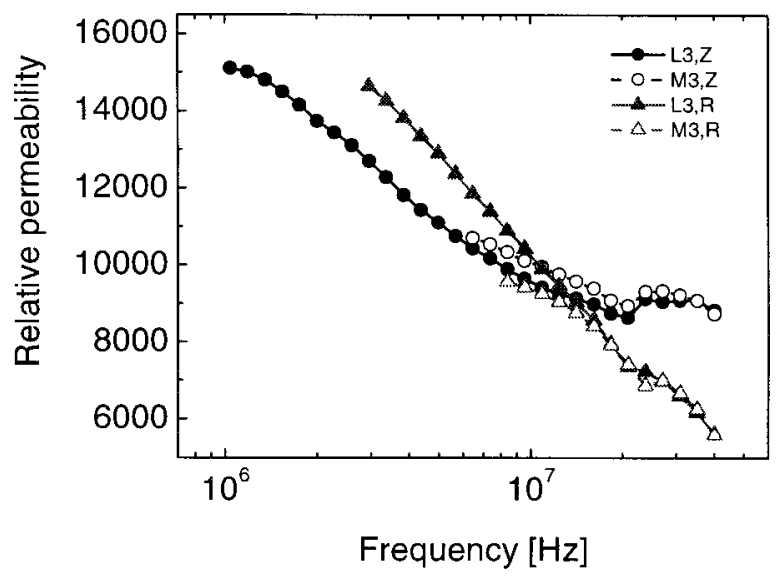

FIG. 3. Frequency variation of the relative permeability of the trilayer structures L3 and M3 of Table I, directly deduced from the resistance $(R)$ and impedance $(Z)$ measurements using the theoretical model of Panina et al. (Ref. 10).

By decreasing the dimensions of the test structures, a much smaller effect is observed. This is clearly evidenced in Figs. 1(a) and 1(b) on samples M3 and S3. The reduction of the GMI and GMR ratios in smaller structures in general seems to be explainable by demagnetizing effects, which become more important with reducing size, and tend to reduce the magnetic flux closure.

Figure 2 shows a three-dimensional plot of the resistance of sample L3 as a function of magnetic field and frequency. With the application of magnetic field, we observe a resistance maximum at around $0.2 \mathrm{mT}$, followed by a sharp decrease with increasing fields. Saturation of the resistance to a lower value takes place at fields around 1-10 mT. Similar graphs to that of Fig. 2 have been obtained, as well as for the impedance, for all the samples studied. We have interpreted our measurements with the theoretical model of Panina and Mohri. ${ }^{10}$ Contrary to our experimental geometry, in this model the conductive and magnetic layers have the same width; for our larger samples, the model is demonstrated to be a reasonable approximation. ${ }^{10}$

Figure 3 shows the frequency-dependent relative permeability, directly derived from the resistance or impedance via relation (1) of Ref. 10. We can derive from both the $Z$ and $R$ data a kind of general trend for the $\mu_{r}$ curve for the Metglas 2714a material, which is consistent with the experimentally observed nearly linear decrease with frequency of the susceptibility of amorphous magnetic materials. ${ }^{13}$

In conclusion, we have studied the giant magnetoimpedance effect in trilayers of micropatterned amorphous magnetic ribbon and $\mathrm{Cu}$ foils. Choosing thick foil for trilayer structures and the Metglas ${ }^{\circledR}$ 2714a material, having an extremely high intrinsic permeability, resulted in very high GMI and GMR values. The high-frequency data are interpreted in terms of the theoretical model of Panina et al., and the high-frequency relative dynamic magnetic permeability for the Metglas 2714a could be determined.

The authors would like to thank F. Vincent for his help with the technology of the test structures and the Swiss National Science Foundation (Grant No. 21-55302.98) for fi- 
${ }^{1}$ K. Mohri, K. Kawashima, T. Kozhawa, Y. Yoshida, and L. V. Panina, IEEE Trans. Magn. 28, 3150 (1992).

${ }^{2}$ K. Mohri, K. Kawashima, T. Kohzawa, and H. Yoshida, IEEE Trans. Magn. 29, 1245 (1993).

${ }^{3}$ F. L. Machado, C. S. Martins, and B. M. Rezende, Phys. Rev. B 51, 3926 (1995).

${ }^{4}$ M. Vázquez and A. Hernando, J. Phys. D 29, 939 (1996).

${ }^{5}$ L. V. Panina, K. Mohri, K. Bushida, and M. Noda, J. Appl. Phys. 76, 6198 (1994).

${ }^{6}$ H. Q. Guo, C. Chen, M. Li, T. Y. Zhao, K. Z. Luan, B. G. Shen, Y. H. Liu, J. G. Zhao, L. M. Mei, and H. Kronmüller, Mater. Sci. Eng., A 226-228, 550 (1997).
${ }^{7}$ H. Q. Guo, H. Kronmüller, T. Dragon, C. Chen, and B. G. Shen, J. Appl. Phys. 84, 5673 (1998).

${ }^{8}$ F. Amalou and M. A. M. Gijs, J. Appl. Phys. 90, 3466 (2001).

${ }^{9}$ S. Xiao, Y. Liu, S. Yan, Y. Dai, L. Zhang, and L. Mei, Phys. Rev. B 61, 5734 (2000)

${ }^{10}$ L. V. Panina and K. Mohri, Sens. Actuators A 81, 71 (2000).

${ }^{11}$ Y. Zhou, J. Yu, X. Zhao, and B. Cai, J. Appl. Phys. 89, 1816 (2001).

${ }^{12}$ Corporate information Honeywell Electronic Materials, URL: http:// www.electronicmaterials.com/about/index.htm

${ }^{13}$ R. Boll, Soft Magnetic Materials. The Vacuumschmelze Handbook (Heyden, London, 1979), pp. 69-79. 